\title{
Lentivirus-mediated downregulation of MAT2B inhibits cell proliferation and induces apoptosis in melanoma
}

\author{
YU LEI ${ }^{1-4}$, BO ZHANG ${ }^{5}$, YAOHUA ZHANG ${ }^{6,7}$, YUAN ZHAO ${ }^{1,2,8}$, \\ JINGYING SUN ${ }^{1-3}$, XUEJUN ZHANG ${ }^{1-3,6}$ and SEN YANG ${ }^{1-3}$
}

\author{
${ }^{1}$ Institute of Dermatology and Department of Dermatology, The First Affiliated Hospital of Anhui Medical University; \\ ${ }^{2}$ Key Laboratory of Dermatology, Anhui Medical University, Ministry of Education of China; \\ ${ }^{3}$ Collaborative Innovation Center for Complex and Severe Skin Diseases, Anhui Medical University; \\ ${ }^{4}$ Department of Oncology, The First Affiliated Hospital of Anhui Medical University, Hefei, Anhui 230022; \\ ${ }^{5}$ Department of Oncology, The Second Affiliated Hospital of Anhui Medical University, Hefei, Anhui 230601; \\ ${ }^{6}$ Institute of Dermatology and ${ }^{7}$ Worldwide Medical Center, Huashan Hospital, Fudan University, Shanghai 200040; \\ ${ }^{8}$ Department of Thoracic Surgery, The First Affiliated Hospital of Anhui Medical University, Hefei, Anhui 230022, P.R. China
}

Received April 18, 2016; Accepted May 31, 2016

DOI: $10.3892 /$ ijo.2016.3603

\begin{abstract}
Malignant melanoma is the most lethal of skin cancers and its pathogenesis is complex and heterogeneous. The efficacy of conventional therapeutic regimens for melanoma remains limited. Thus, it is important to explore novel effective therapeutic targets in the treatment of melanoma. The MAT2B gene encodes for the regulatory subunit of methionine adenosyltransferase (MAT). Recent studies have suggested that MAT2B may have functional roles other than modulating catalytic activity of MAT. In order to identify the roles of MAT2B in the tumorigenesis of malignant melanoma, we compared MAT2B expression profile in melanoma tissues with that in benign nevus samples. We employed lentivirusmediated RNAi to downregulate the expression of MAT2B in malignant melanoma cell lines (A375 and Mel-RM), and investigated the effects of MAT2B on cell growth, colonyformation ability and apoptosis in vitro, as well as tumor growth of a xenograft model in vivo. The expression levels of BCL2 and XAF1 proteins, which were closely related to tumor cell apoptosis, were analyzed by western blot analysis. Our data showed that MAT2B was elevated in both primary and metastatic melanoma tissues compared with benign nevus samples. Lentivirus-mediated downregulation of MAT2B suppressed cell growth, colony formation and induced apoptosis in A375 and Mel-RM cell lines in vitro, affected protein expression
\end{abstract}

Correspondence to: Professor Sen Yang, Institute of Dermatology and Department of Dermatology, The First Affiliated Hospital of Anhui Medical University, No. 218 Jixi Road, Hefei, Anhui 230022, P.R. China

E-mail: yangsen@medmail.com.cn

Key words: MAT2B, melanoma, cell proliferation, apoptosis, BCL2, XAF1 of BCL2 and XAF1, extended the transplanted tumor growth in vivo. These results indicated that MAT2B was critical in the proliferation of melanoma cells and tumorigenicity. It may be considered as a potential anti-melanoma therapeutic target.

\section{Introduction}

Methionine adenosyltransferase (MAT) is a critical enzyme that catalyzes the biosynthesis of S-adenosylmethionine (SAMe), the principal biological methyl donor in all mammalian cells (1). These reactions play a central role in methionine cycle and are associated with several important biological functions such as transmethylation, trans-sulfuration and polyamine synthesis. There are three MAT genes in mammals: two of them (MAT1A and MAT2A) encode for the catalytic subunits (MAT $\alpha 1$ and $\alpha 2$ ) of the different MAT isoforms, while the third gene (MAT2B) encodes for the regulatory subunit (MAT $\beta$ ) which modulate the catalytic activity of MAT. In mammals, three distinct isoforms of MAT (MATI, MATII and MATIII) have been identified: MAT1A, mainly expressed in normal hepatocytes, encodes for MAT $\alpha 1$ which constructs the tetramer (MATI) and the dimer (MATIII); MATII, mainly expressed in extra-hepatic tissues, is a heterotetramer composed by catalytic subunit MAT $\alpha 2$, encoded by MAT2A, and regulatory subunit MAT $\beta$, encoded by MAT2B (2). The MAT $\beta$ subunit regulates the catalytic subunit MAT $\alpha 2$ by reducing the $\mathrm{Km}$ of MATII for methionine and increasing the sensitivity of the enzyme to feedback inhibition by SAMe (3). Downregulation of the MAT $\beta$ subunit expression causes a 6to 10 -fold increase in intracellular SAMe levels (4).

Although MAT2B is known as a gene encoding regulatory subunit for changing MATII enzymatic activity, recent studies have reported that MAT2B also has several kinds of specific biologic fuctions, especially in cancer. MAT2B expression is elevated in human hepatocellular carcinoma and colon cancer which promotes the growth advantage or cell cycle of tumor cells (5-9). In HCC cells, MAT2B knockdown led to growth 
inhibition by downregulating cyclin D1. Moreover, apoptosis of HCC cells induced by LV-shRNA of MAT2B was involved in downregulating BCL-XL and upregulating BCL-XS (6). In both liver and colon cancers, MAT2B interacted with GIT1 and formed a scaffold to regulate Ras/Raf/MEK1/2 activity and therefore promoted cell growth and tumorigenesis $(7,8)$. MAT2B was also identified to interact with HuR, an mRNAbinding protein known to stabilize the mRNA of several cyclins, to affect tumor cell proliferation and apoptosis (9). These data suggest that MAT2B may also have other functional roles in addition to modulating catalytic activity of methionine adenosyltransferase. It may also play a positive role in tumor cell proliferation. Despite several studies supporting the biological function of MAT2B in the tumorigenesis of human hepatocellular carcinoma and colon cancer, its function in the proliferation of cancer cells remains unclear. Moreover, the biofunctions of MAT2B have not been studied in human malignant melanoma.

Malignant melanoma is the most lethal of skin cancers and its pathogenesis is complex and heterogeneous. The incidence of malignant melanoma continues to rise (10). The efficacy of conventional therapeutic regimens for melanoma remains limited, so that it is significant to explore new effective therapies such as targeted therapy in the treatment of melanoma (11). BRAF, NRAS and KIT are three well-known oncogenes associated with malignant melanoma. Several targeted drugs such as vemurafenib and dabrafenib have improved progression-free survival and overall survival in malignant melanoma patients with BRAF V600E mutation, compared with chemotherapy (12-14). However, the recurrences often develop several months later and the tumor usually becomes more resistant to the drug. The subsequent metastasis is even more aggressive and is the critical cause of mortality. These reasons highlight the importance of finding novel therapeutic targets to fight against malignant melanoma. With the development of biological research techniques and methods, further investigation on the molecular pathogenesis of this disease is feasible.

Up to now, the expression of MAT2B in malignant melanoma tissue and its biological functions in melanoma cells has not been clearly demonstrated. Hence, in order to identify the role of MAT2B in malignant melanoma tumorigenesis, we contrast the expression profile of MAT2B in clinical malignant melanoma tissues and benign nevus samples. Furthermore, by using lentivirus-mediated RNAi to downregulate the expression of MAT2B in malignant melanoma cell lines (A375 and Mel-RM), we investigated the effects of MAT2B on cell growth, colony-formation ability and apoptosis in vitro, as well as tumor growth of xengrafts in vivo. Western blot analysis was further carried out to explore the expression patterns of some apoptosis-related proteins.

\section{Materials and methods}

Cell lines, tissue samples and animals. The human melanoma cell line A375 was kindly provided by Shanghai Cell Bank, Chinese Academy of Science. The human melanoma cell line Mel-RM was a gift from Professor Mian Wu (University of Science and Technology of China, Hefei, China). Both cell lines were cultured in Dulbecco's modified Eagle's medium
(DMEM; Gibco-BRL, Grand Island, NY, USA) plus 10\% fetal bovine serum (FBS; HyClone Laboratories, Inc., Logan, UT, USA) in a $5 \% \mathrm{CO}_{2}$ incubator at $37^{\circ} \mathrm{C}$.

Forty-nine primary melanoma tissues, 38 metastatic melanoma tissues and 42 benign nevus samples were obtained from the Department of Surgery, The First and Second Affiliated Hospitals of Anhui Medical University between 2010 and 2015. The histological diagnoses were evaluated by experienced pathologists based on formalin-fixed paraffinembedded (FFPE) tissue sections that were stained with haematoxylin-eosin $(\mathrm{H} \& \mathrm{E})$. Fresh tissue samples were fixed in $4 \%$ paraformaldehyde for $12-24 \mathrm{~h}$ and then paraffin-embedded to test the endogenous expression of MAT2B by immunohistochemistry technique. This study was approved by the Human Research Ethics Committees of the Anhui Medical University and was performed according to the relevant regulations. Written informed consent was obtained from each participant.

The experiments in vivo were licensed by the Animal Research Ethics Committee of Anhui Medical University, Hefei, China, and were conducted following the regulations of the use and care of laboratory animals. Specific pathogenfree (SPF) grade male BALB/c nude mice aged 4 weeks were purchased from Shanghai SLAC laboratory Animal Co., Ltd., Shanghai, China, and were maintained in a $12 \mathrm{~h}$ light/12 h dark cycle at constant temperature.

Immunohistochemistry. Immunohistochemical staining was employed to test the endogenous expression of MAT2B in primary, metastatic melanoma tissues and benign nevus samples. Briefly, formalin-fixed paraffin-embedded tissue sections were deparaffinized using xylene at room temperature, rehydrated through graded ethanol. The sections were then boiled using microwave for $10 \mathrm{~min}$ in citrate buffer for antigen retrieval. Endogenous peroxidase activity was blocked by $3 \%$ hydrogen peroxide for $20 \mathrm{~min}$. The MAT2B antibody (Sigma-Aldrich, St. Louis, MO, USA) was used at a dilution of 1:200, incubated for $12 \mathrm{~h}$ at $4^{\circ} \mathrm{C}$. Finally, the slides were incubated with secondary antibodies (ZSGB-Bio Co., Beijing, China) for $30 \mathrm{~min}$ at $37^{\circ} \mathrm{C}$. The tissue was visualized with 3,3'-diaminobenzidine (DAB) stain and counter-stained for microscopic examination. Two pathologists scored the slides for the staining intensity, independently. The scoring of the slides was conducted blinded to the clinical information. The histological score (H-Score) $(15,16)$ was used to semiquantitative analyze the endogenous expression of MAT2B in tissue samples. The staining intensity was rated as 0 (no staining), 1+ (weak), 2+ (moderate), or 3+ (strong) based on the intensity of the staining pattern. H-Score (range from 0 to 300) was calculated by adding the multiplication of the different staining intensities using the following equation. $\mathrm{H}-\mathrm{Score}=[1 *(\%$ cells $1+)+2 *(\%$ cells $2+)+3 *(\%$ cells $3+)]$.

Lentiviral vector construction and transfection. The most effective MAT2B-targeted small interfering RNA sequence (AGTTCATCACATCATTCAT) and a negative control small interfering RNA sequence (TTCTCCGAACGTGTCACGT) were selected. Short hairpin RNA (shRNA) was synthesized and was cloned into the pGCSIL-GFP vector (GeneChem Co., Ltd., Shanghai, China) to construct the recombinant plasmid. The pGCSIL-GFP vector contains AgeI/EcoRI enzyme 
cleavage sites and green fluorescent protein (GFP) reporter gene with an internal CMV (cytomegalovirus) promoter. The MAT2B-shRNA recombinant plasmid and assistant packaging plasmid (GeneChem) were co-transfected into HEK293T cells. Lentivirus particles expressing MAT2B-shRNA were collected from the supernate of the culture medium, purified by centrifugal ultrafiltration and stored at $-80^{\circ} \mathrm{C}$.

Cells of human melanoma cell lines A375 and Mel-RM were seeded in 6-well plates and allowed to grow until the density of cells reached $\sim 40 \%$. Appropriate amount of lentivirus were added into the culture medium according to the multiplicity of infection (MOI) which was determined as 20 . Culture medium was refreshed $24 \mathrm{~h}$ after lentivirus infection. GFP fluorescence expression was detected for infecting efficiency evaluation $72 \mathrm{~h}$ after infection by fluorescence microscopy.

Quantitative real-time $P C R$ ( $q R T-P C R)$. The quantitative realtime PCR was carried out to detect the mRNA level of MAT2B in human melanoma cell lines A375 and Mel-RM after infected by lentivirus. Cells were divided into 3 groups: mock (melanoma cells without lentivirus infection), shCtrl (infected melanoma cells by lentivirus expressing non-silencing shRNA) and shMAT2B (infected melanoma cells by lentivirus expressing MAT2B-shRNA). Seventy-two hours after lentivirus infection, the cells were collected and total RNA was extracted by TRIzol (Invitrogen Corp., Carlsbad, CA, USA). According to the instructions of the manufacturer, total RNA was reversely transcripted to cDNA by M-MLV reverse transcriptase kits (Promega, Madison, WI, USA). The SYBR-Green Master Mix kit (Takara Bio Inc., Shiga, Japan) was used, and quntitative real-time PCR was performed on Agilent Mx3000P QPCR system (Agilent Technologies, Santa Clara, CA, USA). The following primers were used: MAT2B forward primer 5'-ACAGAGAGGAAGACATACCAG-3' and reverse primer 5'-GTTCATTGCCAGACCAGTG-3'; GAPDH forward primer 5'-TGACTTCAACAGCGACACCCA-3' and reverse primer 5'-CACCCTGTTGCTGTAGCCAAA-3'. The cycling profile was: initial denaturation at $95^{\circ} \mathrm{C}$ for $15 \mathrm{sec}, 45$ cycles consisting of $95^{\circ} \mathrm{C}$ for $5 \mathrm{sec}, 60^{\circ} \mathrm{C}$ for $30 \mathrm{sec}$. Data were calculated using the $2^{-\Delta \Delta \mathrm{CT}}$ method with GAPDH serving as an internal control. All experiments were independently repeated at least 3 times.

Cell proliferation assay. Two methods were carried out to measure the cell proliferation of human melanoma cells after lentivirus infection. First, cell growth of human melanoma cell line A375 was measured by the fluorescent cytometer. A375 cells were infected with shCtrl or shMAT2B lentivirus. Seventy-two hours after infection, cells were collected, counted and seeded into 96-well plates. All cells with green fluorescence were counted by Nexcelom Celigo Image Cytometer in the following 5 days. Fluorescent photomicrographs were taken and cell growth curves were drawn. Second, in order to confirm the inhibition effect and test whether the shCtrl lentivirus can affect cell proliferation, we conducted the MTT assay in human melanoma cell lines A375 and Mel-RM. Cells were divided into 3 groups as described above: Mock, shCtrl and shMAT2B. Seventy-two hours after lentivirus infection, cells were collected and seeded in 96-well plates. MTT (Sigma-Aldrich) solution was added after 24, 48, 72,
96 and $120 \mathrm{~h}$. The cells were then cultured for another $4 \mathrm{~h}$. The resulting formazan crystals were dissolved in DMSO and absorbance at $490 \mathrm{~nm}$ was detected. The experiments were repeated at least three times.

Colony formation assay. The colony-forming abilities of human melanoma cell lines A375 and Mel-RM were tested. Cells were divided into 3 groups: Mock, shCtrl and shMAT2B, as described above. Seventy-two hours after lentivirus infection, cells at the logarithmic phase were collected and inoculated at a density of 300 cells/well in 6-well plates. Culture medium was refreshed every 3 days. After incubating for 14 days, cells were fixed with $4 \%$ paraformaldehyde and stained with Giemsa. Cell colonies that contained at least 50 cells were manually counted.

Apoptosis assay. The apoptosis ratio of human melanoma cell lines A375 and Mel-RM was detected by a flow cytometer. As described above, cells in Mock group were not infected by any lentivirus, cells in shCtrl or shMAT2B group were infected by lentivirus expressing non-silencing shRNA or MAT2B-shRNA. Seventy-two hours after lentivirus infection, melanoma cells were collected, washed and then stained by Annexin V-APC at room temperature in the dark for $15 \mathrm{~min}$. After filtered by a $50 \mu \mathrm{m}$ mesh, apoptosis ratio of each sample was detected by Millipore Guava easyCyte HT flow cytometer (Millipore, Billerica, MA, USA).

Western blot analysis. Western blot analysis was conducted to measure the variations in protein expression after MAT2B downregulation in human melanoma cell lines A375 and Mel-RM. Cells were divided into 3 groups: Mock, shCtrl and shMAT2B. Seventy-two hours after lentivirus infection, cells were collected. Proteins were extracted, loaded, separated by SDS polyacrylamide gel electrophoresis and transferred onto a PVDF (polyvinylidene difluoride) membrane. The membrane was blocked, washed and incubated overnight with primary antibodies against MAT2B (1:500 dilution; Sigma-Aldrich), XAF1 (1:1,000 dilution; Santa Cruz Biotechnology, Dallas, TX, USA), BCL2 (1:1,500 dilution; Santa Cruz Biotechnology). After that, the membrane was washed and incubated with secondary antibodies (Santa Cruz Biotechnology) and detected by enhanced chemiluminescence. The intensity of bands was quantified by Image J software and protein expression was standardized to GAPDH.

Tumor growth experiment in vivo. For the transplanted tumor model, A375 cells were infected by shMAT2B or shCtrl lentivirus. After lentivirus infection, cells at the logarithmic phase were collected and adjusted at a density of $2 \times 10^{7}$ cells $/ \mathrm{ml}$. The cell suspension $(250 \mu \mathrm{l})$ was subcutaneously injected into the right dorsal flank of the nude mice. After implantation, tumors were allowed to grow until their diameters reached $\sim 5 \mathrm{~mm}$. Tumor size was measured every 4 days and tumor volumes were calculated using the formula: $V=\left(\pi a b^{2}\right) / 6$, where a was the largest superficial diameter and $b$ was the perpendicular diameter. Twenty-four days later, the mice were sacrificed and the tumors were dissected and weighted. Inhibition ratio was defined as 1 - (tumor weight of shMAT2B group/shCtrl group). As previously described (17), relative tumor volume was used to 

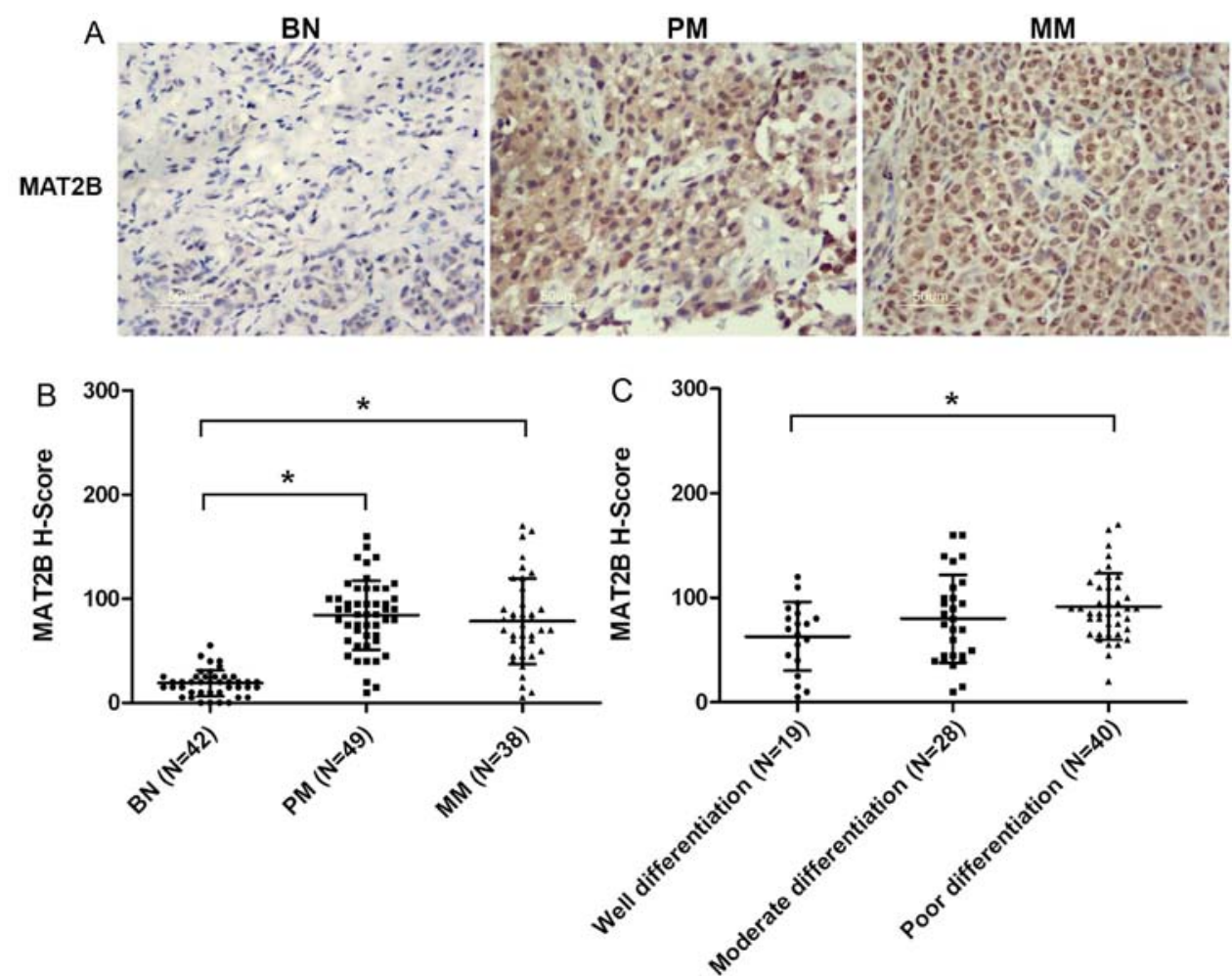

Figure 1. MAT2B expression was upregulated in 49 primary melanoma (PM) and 38 metastatic melanoma (MM) tissues compared to 42 benign nevus (BN) samples and was associated with melanoma differentiation status. (A) Representative microphotographs of MAT2B protein immunohistochemistry staining (magnification, x400). (B) MAT2B protein expressions in melanoma tissues and nevus. (C) MAT2B protein expressions with tumor differentiation status. Data are presented as mean histological score $(\mathrm{H}-\mathrm{Score}) \pm \mathrm{SD}$. Statistical comparisons were performed by Kruskal-Wallis test. ${ }^{*} \mathrm{P}<0.05$.

create the tumor growth curve and it was regarded as the ratio of measured tumor volume compared with the initial tumor volume of the same mouse. Tumor growth equations were constructed based on the Gompertz model, which was determined by the equation: $\mathrm{y}=\mathrm{V}_{0} * \exp \{\mathrm{b} *[1-\exp (-\mathrm{a} * \mathrm{x})]\}$, where $\mathrm{y}$ was tumor size, $\mathrm{x}$ was time, $\mathrm{V}_{0}$ was the initial tumor volume, $\mathrm{a}$ and $\mathrm{b}$ were constant (18). Tumor growth time (TGT 10) was the number of days it took the tumor to grow to 10 times the size of its initial volume. Tumor growth delay (TGD) was regarded as the time difference of TGT 10 between shMAT2B group and shCtrl group.

Statistical analysis. Quantitative data are presented as the mean \pm standard deviation of three independent experiments. Statistical analysis was performed by SPSS 13.0 software. Statistical comparisons were performed by two-tailed t-test or one-way analysis of variance for parameter test. The expression of MAT2B in primary melanoma tissues, metastatic melanoma tissues and nevi tissues was compared by KruskalWallis test. Comparisons of tumor growth curves in vivo were completed by repeated-measures variance analysis. A P-value $<0.05$ was considered as statistically significant.

\section{Results}

MAT2B expression is frequently upregulated in malignant melanoma tissues. We examined the endogenous expression of MAT2B by conducting immunohistochemical staining on formalin-fixed paraffin-embedded samples from 49 patients with primary melanoma, 38 patients with metastatic melanoma and 42 patients with benign nevus. As shown in Fig. 1A and B, endogenous expression of MAT2B was elevated in malignant melanoma compared to benign nevus. However, there was no significant difference in MAT2B level between primary and metastatic melanoma. Hierarchical analysis of MAT2B expression in melanoma tissue found that poorly differentiated tumors frequently have higher level of MAT2B compared with well differentiated tumors (Fig. 1C). However, there were no significant changes in MAT2B expression within different gender, age and primary site (data not shown). These results indicated that the expression of MAT2B may be related to the tumorigenesis and differentiation of melanoma.

MAT2B is effectively knocked down in human melanoma cells by MAT2B-shRNA lentivirus. MAT2B-shRNA (shMAT2B) lentivirus and non-silencing shRNA (shCtrl) lentivirus were constructed and infected in human melanoma cell lines A375 and Mel-RM. As shown in Fig. 2A, the infection efficiency was $>90 \%$ according to the GFP expression, $72 \mathrm{~h}$ after lentivirus infection. Quantitative real-time PCR (Fig. 2B) indicated that the MAT2B mRNA level was downregulated $\sim 70-80 \%$ in two cell lines after shMAT2B lentivirus infection. There was no statistical difference in the mRNA level between Mock and shCtrl group. In terms of protein levels, western blot analysis (Fig. 2C and D) exhibited a similar profile. These data suggested that MAT2B was effectively knocked down in human melanoma cell lines A375 and Mel-RM after shMAT2B lentivirus infected, and that shCtrl lentivirus did not change the mRNA or protein level of MAT2B in melanoma cells. 


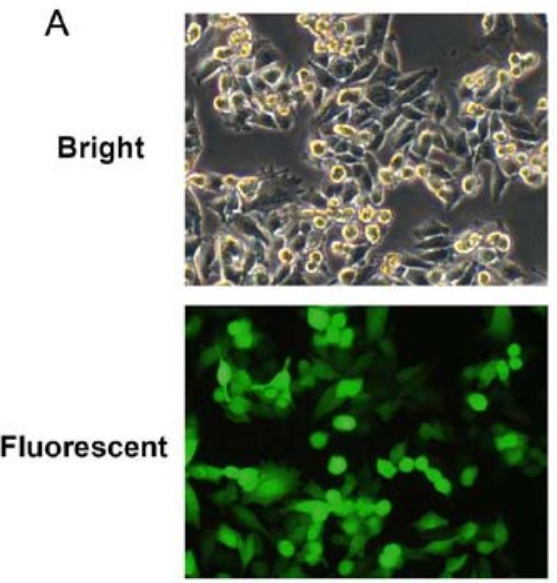

A375
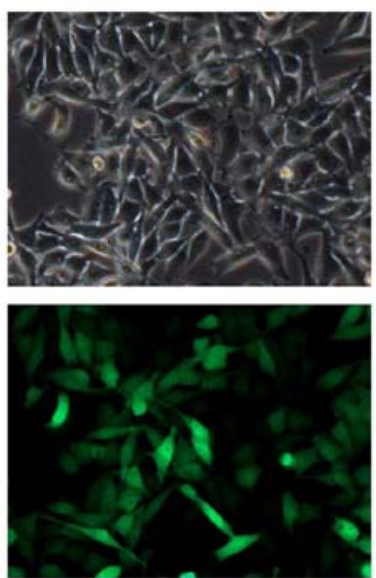

Mel-RM

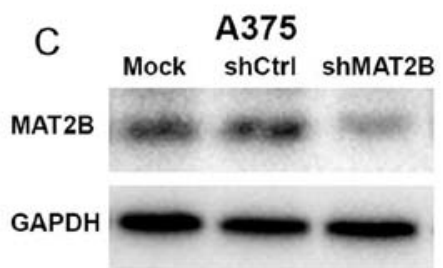

\section{Mel-RM}

Mock shCtrl shMAT2B

\section{MAT2B}

GAPDH
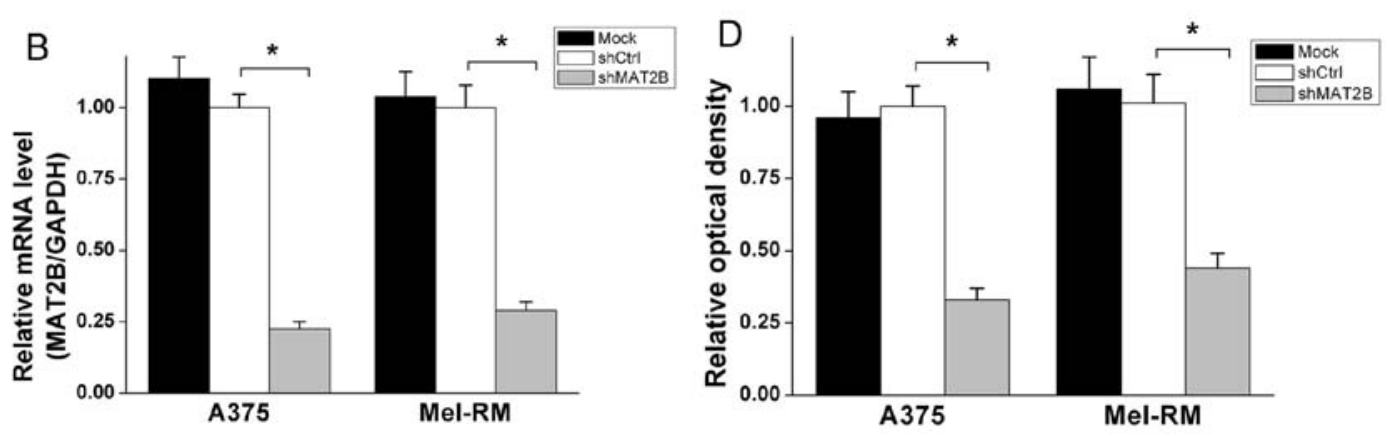

Figure 2. MAT2B is effectively knocked down in human melanoma cells by MAT2B-shRNA lentivirus. (A) Bright and fluorescent photomicrographs (magnification, x200) of human melanoma cell lines A375 and Mel-RM $72 \mathrm{~h}$ after lentivirus infection. (B) MAT2B mRNA level was detected by quantitative real-time PCR $72 \mathrm{~h}$ after lentivirus infection. (C and D), MAT2B protein level (bands and relative optical density) was detected by western blot analysis $72 \mathrm{~h}$ after lentivirus infection. Data are presented as the mean $\pm \mathrm{SD}$ from three independent experiments. ${ }^{*} \mathrm{P}<0.05$.

A

shCtrl

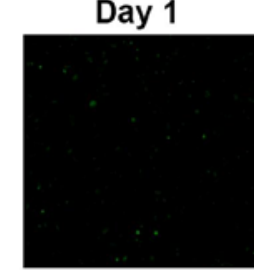

ShMAT2B

B

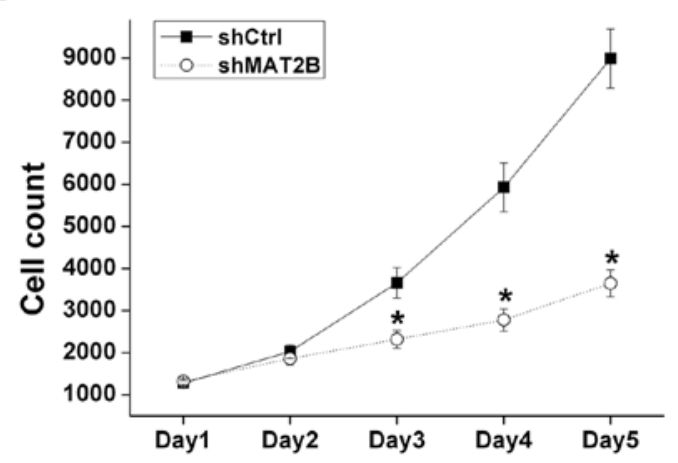

Day 3
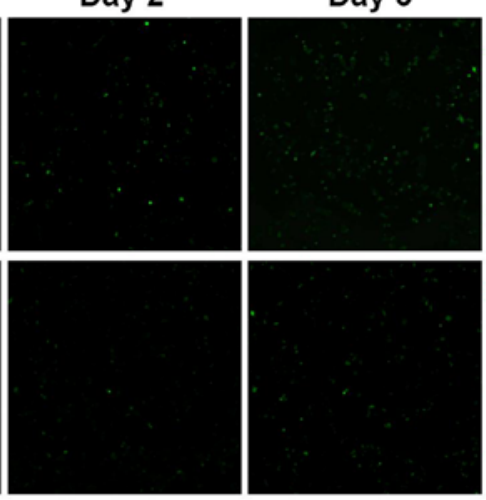

C
Day 4
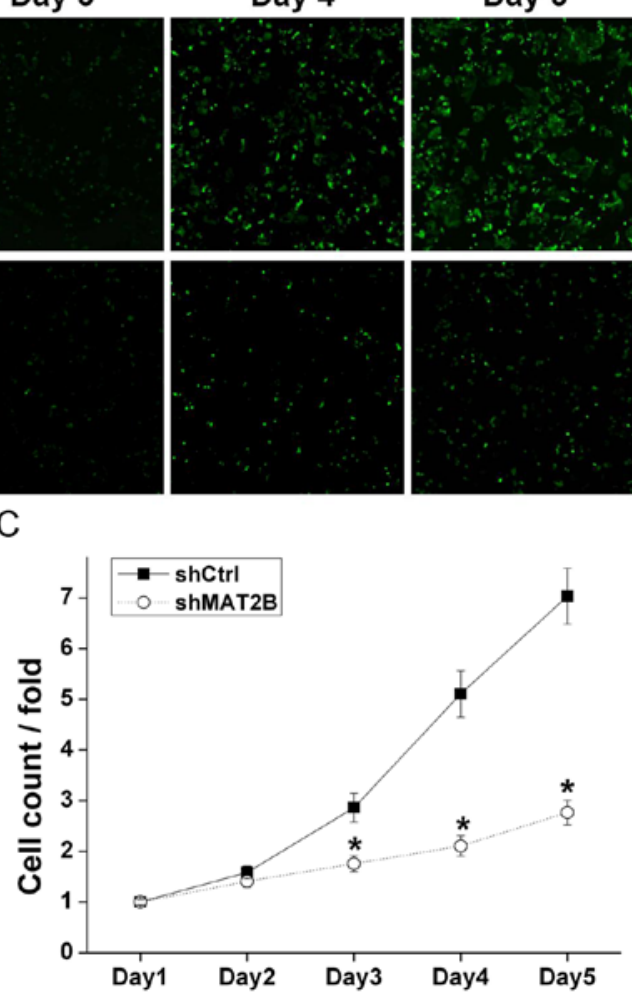

Figure 3. Cell proliferation of A375 is inhibited after shMAT2B lentivirus infection. (A) Fluorescent photomicrographs taken by Nexcelom Celigo Image Cytometer in 5 continuous days. (B) Cell growth curve. (C) Curve of cell count/fold. Data are presented as the mean \pm SD from three independent experiments. ${ }^{*} \mathrm{P}<0.05$. 

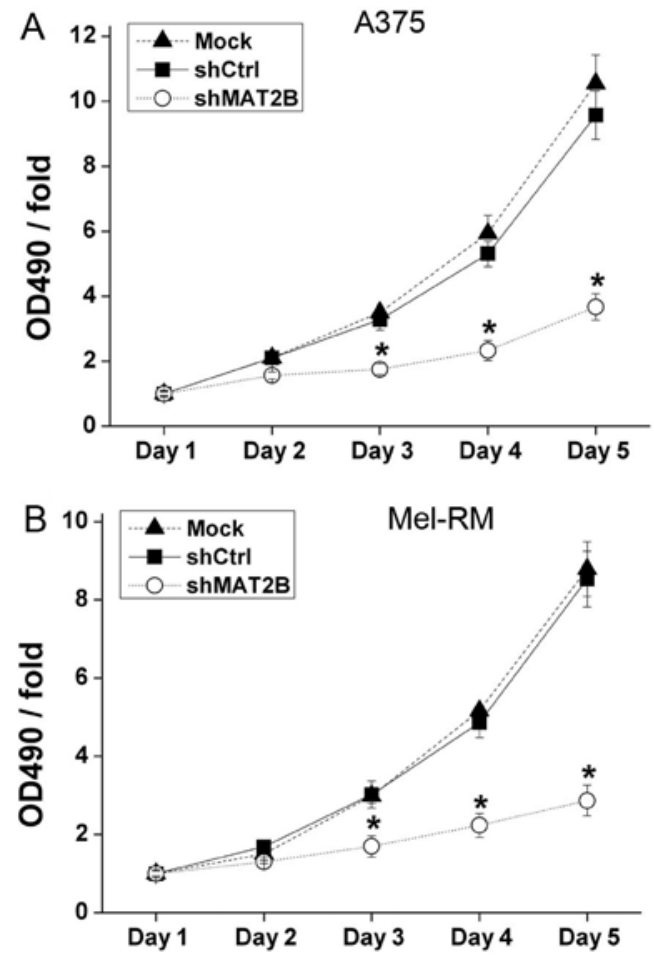

Figure 4. Downregulation of MAT2B inhibited melanoma cell proliferation analyzed by MTT assay. The OD $490 \mathrm{~nm} /$ fold on day 3-5 showed significant differences between shCtrl and shMAT2B groups in melanoma cell lines (A) A375 and (B) Mel-RM, respectively. Data are presented as the mean \pm SD from three independent experiments. $\mathrm{P}<0.05$.

Cell proliferation is inhibited after MAT2B downregulation in human melanoma cells. A375 cells with green fluorescence were counted by Nexcelom Celigo Image Cytometer in continuous 5 days (Fig. 3A) and the cell growth curves were drawn (Fig. 3B and C). After a 5-day culture, A375 cells in shCtrl and shMAT2B groups increased by 7.03 and 2.77 times, respectively, revealing that cell growth of A375 was inhibited after shMAT2B lentivirus infection.

Moreover, in order to confirm the inhibition effect and to detect whether the shCtrl lentivirus can affect cell proliferation, MTT assays were conducted in A375 and Mel-RM, and Mock groups were also added (Fig. 4). The OD $490 \mathrm{~nm} /$ fold on days 3-5 showed significant differences between shCtrl and shMAT2B groups which suggested that downregulation of MAT2B inhibited melanoma cell proliferation. There was no significant change in the OD $490 \mathrm{~nm} /$ fold between Mock and shCtrl groups which confirmed that shCtrl lentivirus infection could not affect cell proliferation.

Colony formation ability is depressed after MAT2B downregulation in human melanoma cells. Colony formation assay was conducted to test the colony formation ability of human melanoma cell lines A375 and Mel-RM after lentivirus infection in vitro. Data are shown in Fig. 5. The number of colonies was significant decreased in shMAT2B group compared with shCtrl group in both A375 and Mel-RM cells. Another interesting result was that shCtrl lentivirus infection inhibited the colony formation ability in A375 cells compared with Mock group. However, the inhibition effect in
A

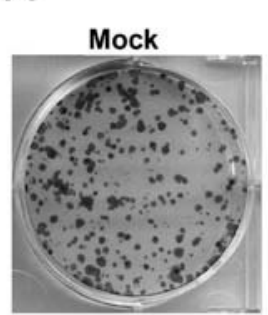

A375

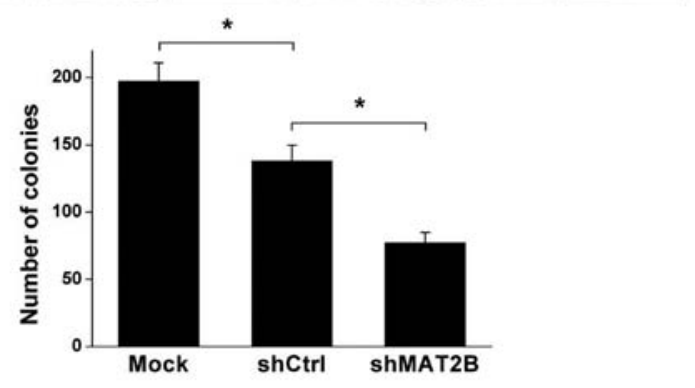

B

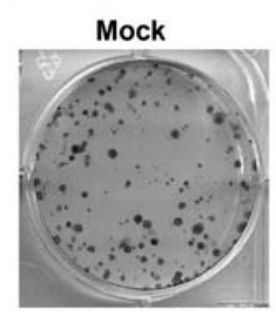

Mel-RM
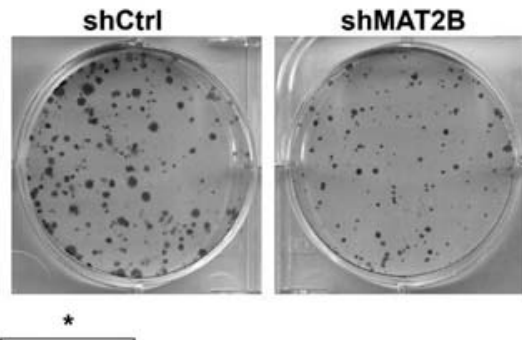

shCtrl
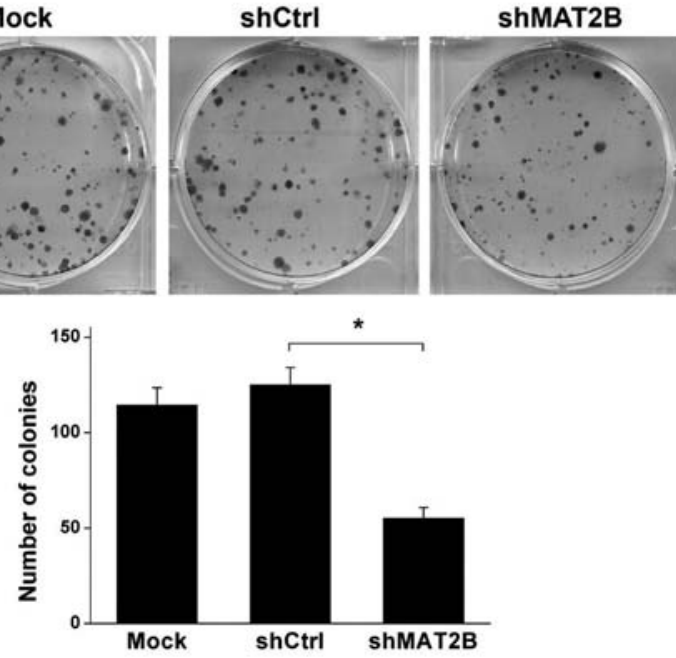

Figure 5. Downregulation of MAT2B depressed colony formation ability of human melanoma cell lines A375 (A) and Mel-RM (B). Colonies of human melanoma A375 and Mel-RM cells in 6-well plates were stained by Giemsa and were quantitatively analyzed. Cell colonies which contained at least 50 cells were counted. Data are presented as the mean \pm SD from three independent experiments. ${ }^{*} \mathrm{P}<0.05$.

Mel-RM cells was not shown as in A375 cells. These results suggested that despite the difference in virus susceptibility of the 2 cell lines, downregulation of MAT2B depressed colony formation ability of A375 and Mel-RM in vitro.

Apoptosis ratio is increased after MAT2B downregulation in human melanoma cells. The apoptosis ratio of each sample was detected with Annexin V-APC staining by a flow cytometer. The results were shown in Fig. 6. The apoptosis ratios of A375 and Mel-RM in shMAT2B group were increased compared with those in shCtrl group, from 4.45 to 18.07 and 4.66 to $11.89 \%$, respectively. Furthermore, there were no significant differences in the apoptosis ratios of these two cell lines between Mock and shCtrl groups. This suggested that MAT2B knockdown promoted apoptosis of human melanoma cells in vitro. 
A
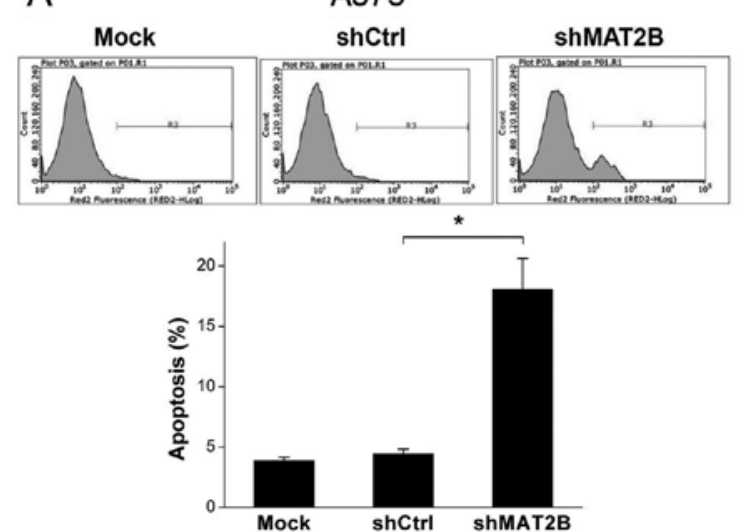

B
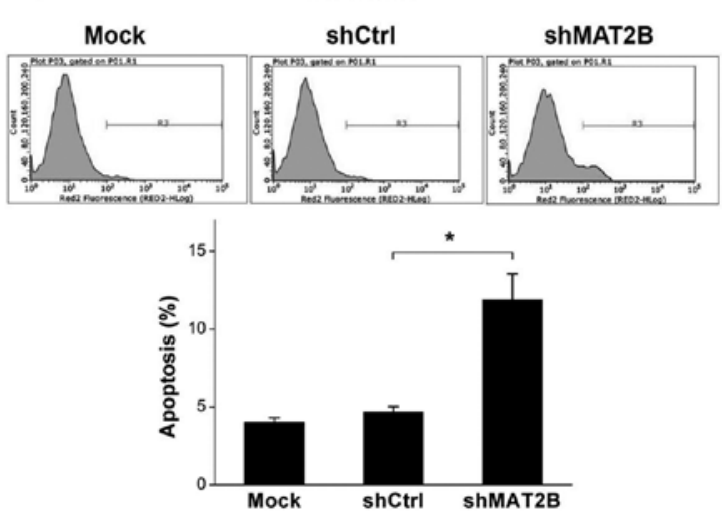

Figure 6. MAT2B knockdown promotes apoptosis of human melanoma cell lines A375 (A) and Mel-RM (B). Flow cytometric analysis displayed that the apoptosis ratios of these two cell lines in shMAT2B group were increased compared with those in shCtrl group, respectively. Data are presented as the mean \pm SD from three independent experiments. ${ }^{*} \mathrm{P}<0.05$.

A

MAT2B

XAF1

BCL2
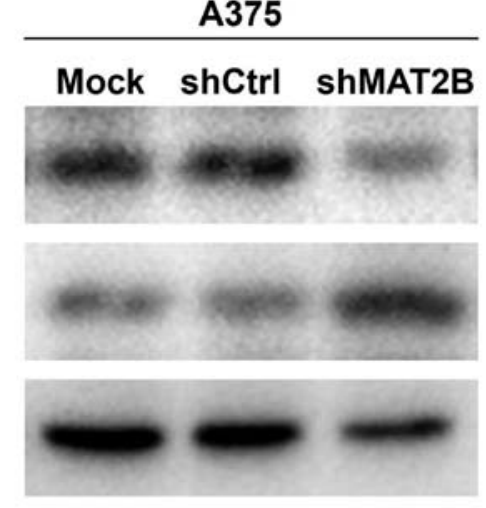

\section{GAPDH}

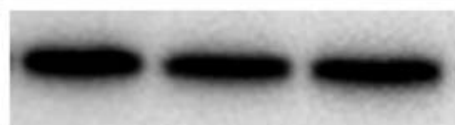

Mel-RM
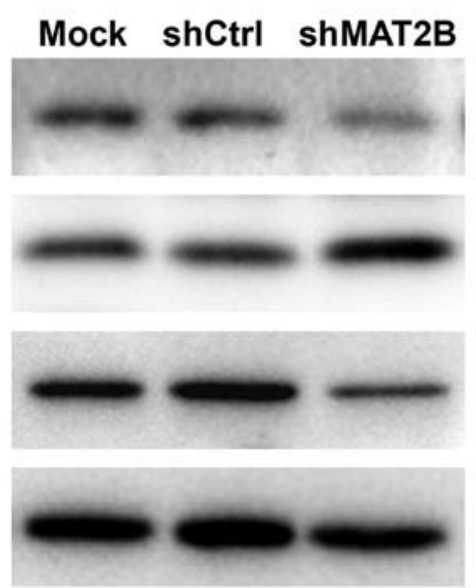

B
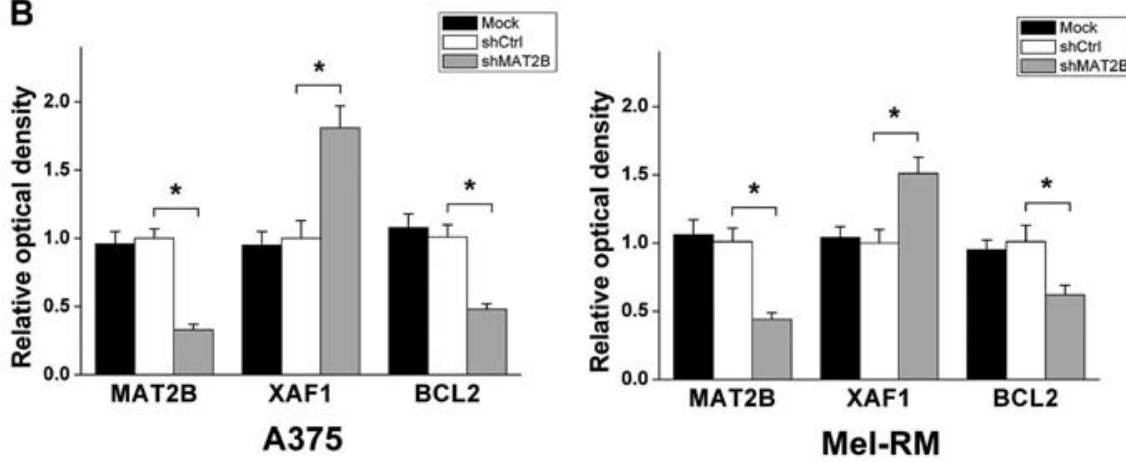

Figure 7. Protein expression levels of BCL2 and XAF1, which were closely related to apoptosis of tumor cells, were analyzed by western blot analysis (A) Protein levels of MAT2B, BCL2 and XAF1 were analyzed by western blot analysis. (B) The relative optical density of each band was quantified by ImageJ software and standardized to GAPDH. Data are presented as the mean \pm SD from three independent experiments. $\mathrm{P}<0.05$.

Protein expression levels of BCL2 and XAF1 are significantly changed after MAT2B downregulation in human melanoma cells. Protein expression levels of BCL2 and XAF1, which were closely related to apoptosis of tumor cells, were analyzed by western blot analysis. As shown in Fig. 7, XAF1 was significantly upregulated while BCL2 protein expression was significantly downregulated after MAT2B was knocked down in human melanoma cell lines A375 and Mel-RM.
MAT2B downregulation delays tumor growth in vivo. To investigate the effect of MAT2B on tumor growth in vivo, a transplanted tumor model was established by subcutaneously injecting A375 (infected by shCtrl or shMAT2B lentivirus) into the right dorsal flank of the BALB/c nude mice. Twentyfour days later, tumors were dissected and weighed, the shMAT2B group formed smaller and lighter tumors than the shCtrl group (Fig. 8A, C and D). Tumor inhibition ratio was 

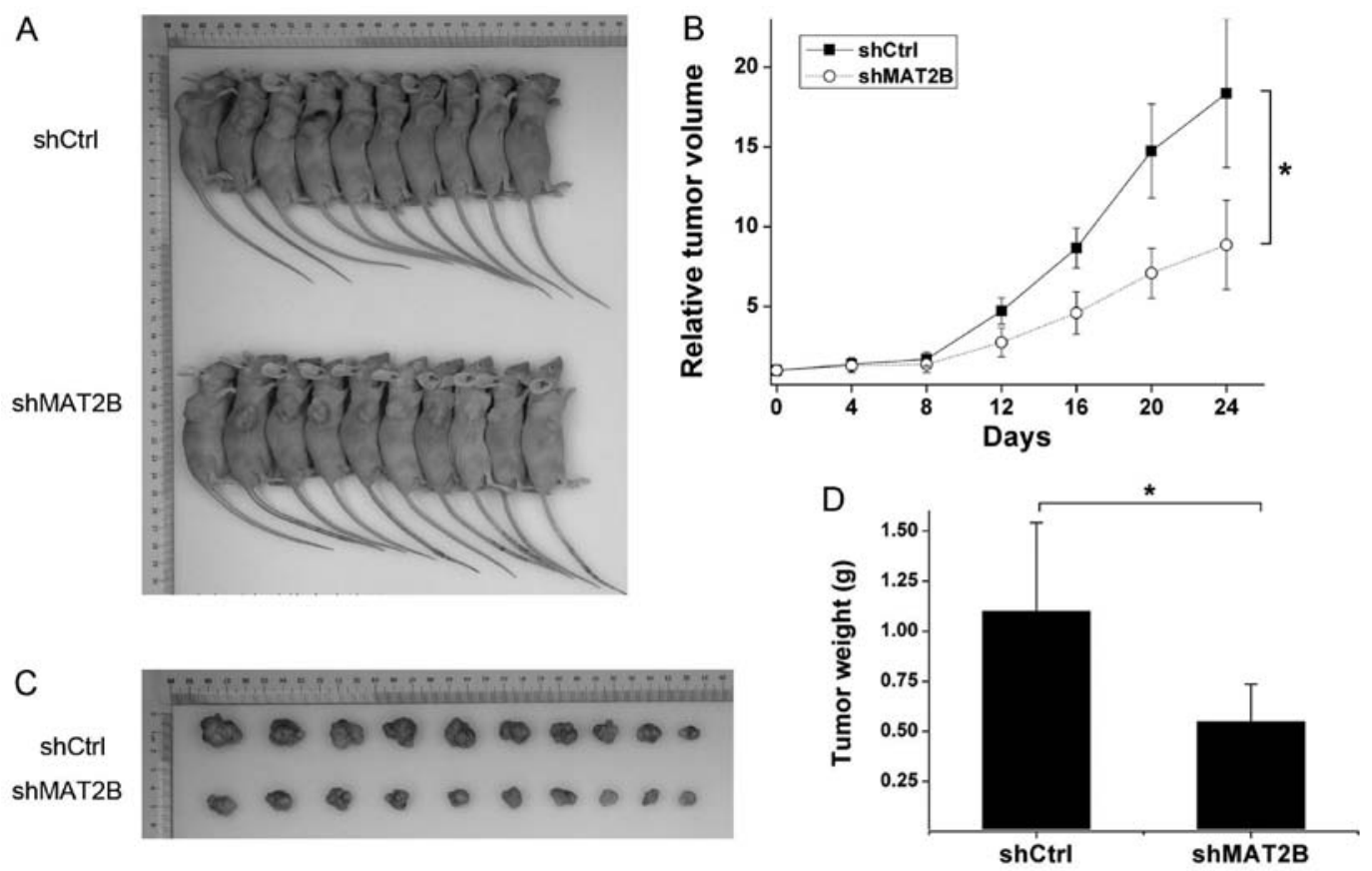

Figure 8. MAT2B knockdown inhibits the transplanted tumor growth in vivo. A375 infected by shCtrl or shMAT2B lentivirus were subcutaneously injected into the right dorsal flank of the BALB/c nude mice to establish the experimental model. (A) Twenty-four days later, shMAT2B group had formed smaller tumors than shCtrl group. (B) Tumor growth curves. (C) Pictures of the excised tumors. (D) Tumor weight. Statistical comparisons of tumor growth were performed by repeated-measures variance analysis. ${ }^{\mathrm{P}}<0.05$.

$50.09 \%$. Tumor growth curves are shown in Fig. 8B. Tumor grow th equations were constructed according to the Gompertz model and used to calculate parameters such as tumor growth time (TGT 10) and tumor growth delay (TGD). The TGT 10 was 17.05 and 27.76 days in shCtrl and shMAT2B groups, respectively. TGD was 10.71 days between the two groups. Through comparing tumor growth between shCtrl and shMAT2B groups by repeated-measures variance analysis, we observed that downregulation of MAT2B extended the transplanted tumor growth in vivo.

\section{Discussion}

Recent studies have suggested that MAT2B may also have other functional roles in addition to modulating catalytic activity of methionine adenosyltransferase. In the present study, in order to explore the expression profiles and biological functions of MAT2B in human malignant melanoma, we investigated the expression profiles of MAT2B in primary and metastatic malignant melanoma compared with benign nevus by immunohistochemistry technique, and selected lentivirus-mediated RNAi for MAT2B downregulation. We found that MAT2B was expressed at higher levels in primary and metastatic melanoma tissues compared with nevus. Lentivirus-mediated downregulation of MAT2B suppressed cell growth and colony formation abilities, enhanced apoptosis in human malignant melanoma cell lines A375 and Mel-RM in vitro. In addition, we provided evidence that downregulation of MAT2B inhibited tumor growth of xenografts in vivo. Then protein expression of BCL2 and XAF1, which were closely related to apoptosis of tumor cells, were analyzed by western blot analysis. The results implied that some of the pro-apoptotic genes were upgraded and anti-apoptotic genes downgraded after MAT2B knocked down in human melanoma cell lines A375 and Mel-RM.

Apoptosis plays a crucial role in maintaining proper tissue development and homeostasis. Apoptosis escaping by cancer cells is a strategy of their adaption to microenvironment and treatment (19). Anti-apoptotic molecules can protect tumor cells from apoptosis and mediate some other processes, combination with lacking of pro-apoptotic molecules, result in an enhanced aggressive phenotype $(20,21)$.

The BCL2 family is considered as the most important and potent mediators of apoptosis and survival in human cancers including melanoma (22). Since it was discovered in the late 1980s, their mechanisms of action and interactions in apoptosis have been elucidated in exquisite molecular detail (23). BCL2 is the core member of the family with the functions of promoting cell survival and inhibiting apoptosis. The BCL2 anti-apoptotic gene is overexpressed in many human cancers, and leads to aggressive disease course and poor survival in patients with different cancers. This overexpression can result from chromosomal translocations, gene amplification, increased gene transcription, altered post-translational processing and promoter hypomethylation (24). In the present study, the expression level of BCL2 anti-apoptotic gene was attenuated after MAT2B downregulation which induced apoptosis and inhibited cell proliferation both in vitro and in vivo.

$\mathrm{X}$-linked inhibitor of apoptosis protein (XIAP) is the most potent member in the inhibitors of apoptosis (IAP) family. It is well known as an inhibitor of apoptosis by binding to caspase-3, -7 and -9 to suppress their activities (25). XIAP-associated factor 1 (XAF1) was identified as a XIAP-binding protein and could directly bind preferentially to XIAP and antagonize the anti-caspase activity of XIAP to induce apoptosis (26). 
Pro-apoptotic gene XAF1 was constantly expressed in normal and fetal tissues but weakly expressed in most human cancer cell lines and human cancer tissues such as ovarian, hepatocellular, colon, pancreatic cancer and melanoma (27-31). Recent studies had shown that XAF1 expression was relatively low in hepatocellular carcinoma cell lines and hepatoma tissues. Adenovirus-mediated XAF1 overexpression inhibited cell proliferation and induced apoptosis, and prolonged the survival of tumor-bearing mice, suggested that XAF1 could be associated with tumor growth by inducing apoptosis and inhibiting cell proliferation (28). In the present study, the proapoptotic gene XAF1 expression was significantly augmented after MAT2B downregulation, then lead to similar tumordepressing functions in melanoma cell lines in vitro and A375 transplanted tumor model in vivo.

Another interesting result was observed in colony formation assay. The number of colonies was significant decreased in shCtrl group compared with Mock group in A375 cells. Suggesting that shCtrl lentivirus infection inhibited the colony formation ability in A375, but it did not show the same inhibition effect in Mel-RM cells in colony formation assay. In MTT assay, however, the OD $490 \mathrm{~nm} /$ fold showed no significant difference between Mock and shCtrl groups in both A375 and Mel-RM cells, since the cell density was different between colony-forming assay and MTT assay. These results implied that, different cell lines have various susceptibilities to virus infection, and possibly A375 in a low density would become more sensitive to virus infection.

In the present study, we only used single shRNA sequence to detect the bio-functions of MAT2B. It may bring out the offtarget effects of RNA interference. In order to eliminate the off-target effects, we selected two malignant melanoma cell lines to confirm the functional results because these effects were probably different between A375 and Mel-RM cell lines. Employing multiple shRNA sequences in RNA interference may present stricter results.

In conclusion, our data showed that MAT2B expression was elevated in malignant melanoma compared with benign nevus and was associated with tumor differentiation status. Knockdown of MAT2B suppressed cell growth, colony formation ability and induced apoptosis in vitro, as well as extended the transplanted tumor growth in vivo. The possible mechanisms could be associated with the upregulation of some pro-apoptotic genes expression such as XAF1 and downregulation of some anti-apoptotic genes expression such as BCL2. However, the particular mechanisms still need to be confirmed. These results indicated that MAT2B was critical for melanoma cell proliferation and tumorigenicity. It is a probable target of anti-melanoma therapy.

\section{Acknowledgements}

The present study is supported by the Fund for Program of Collaborative Innovation Center for Complex and Severe Skin Diseases, Anhui Medical University (4601001116).

\section{References}

1. Lu SC and Mato JM: S-adenosylmethionine in liver health, injury, and cancer. Physiol Rev 92: 1515-1542, 2012.
2. Halim AB, LeGros L, Geller A and Kotb M: Expression and functional interaction of the catalytic and regulatory subunits of human methionine adenosyltransferase in mammalian cells. J Biol Chem 274: 29720-29725, 1999.

3. LeGros HL Jr, Halim AB, Geller AM and Kotb M: Cloning, expression, and functional characterization of the $\beta$ regulatory subunit of human methionine adenosyltransferase (MAT II). J Biol Chem 275: 2359-2366, 2000.

4. LeGros L, Halim AB, Chamberlin ME, Geller A and Kotb M: Regulation of the human MAT2B gene encoding the regulatory beta subunit of methionine adenosyltransferase, MAT II. J Biol Chem 276: 24918-24924, 2001.

5. Yang H, Ara AI, Magilnick N, Xia M, Ramani K, Chen H, Lee TD, Mato JM and Lu SC: Expression pattern, regulation, and functions of methionine adenosyltransferase 2beta splicing variants in hepatoma cells. Gastroenterology 134: 281-291, 2008.

6. Wang Q, Liu QY, Liu ZS, Qian Q, Sun Q and Pan DY: Lentivirus mediated shRNA interference targeting MAT2B induces growth-inhibition and apoptosis in hepatocelluar carcinoma. World J Gastroenterol 14: 4633-4642, 2008.

7. Peng H, Dara L, Li TW, Zheng Y, Yang H, Tomasi ML, Tomasi I, Giordano P, Mato JM and Lu SC: MAT2B-GIT1 interplay activates MEK1/ERK 1 and 2 to induce growth in human liver and colon cancer. Hepatology 57: 2299-2313, 2013.

8. Peng H, Li TW, Yang H, Moyer MP, Mato JM and Lu SC: Methionine adenosyltransferase 2B-GIT1 complex serves as a scaffold to regulate Ras/Raf/MEK1/2 activity in human liver and colon cancer cells. Am J Pathol 185: 1135-1144, 2015.

9. Xia M, Chen Y, Wang LC, Zandi E, Yang H, Bemanian S, Martínez-Chantar ML, Mato JM and Lu SC: Novel function and intracellular localization of methionine adenosyltransferase 2beta splicing variants. J Biol Chem 285: 20015-20021, 2010.

10. Siegel RL, Miller KD and Jemal A: Cancer statistics, 2015. CA Cancer J Clin 65: 5-29, 2015.

11. Russo A, Ficili B, Candido S, Pezzino FM, Guarneri C, Biondi A, Travali S, McCubrey JA, Spandidos DA and Libra M: Emerging targeted therapies for melanoma treatment (Review). Int J Oncol 45: 516-524, 2014.

12. Wilmott JS, Menzies AM, Haydu LE, Capper D, Preusser M, Zhang YE, Thompson JF, Kefford RF, von Deimling A, Scolyer RA, et al: BRAF(V600E) protein expression and outcome from BRAF inhibitor treatment in BRAF(V600E) metastatic melanoma. Br J Cancer 108: 924-931, 2013.

13. Sosman JA, Kim KB, Schuchter L, Gonzalez R, Pavlick AC, Weber JS, McArthur GA, Hutson TE, Moschos SJ, Flaherty KT, et al: Survival in BRAF V600-mutant advanced melanoma treated with vemurafenib. N Engl J Med 366: 707-714, 2012.

14. Ascierto PA, Minor D, Ribas A, Lebbe C, O'Hagan A, Arya N, Guckert M, Schadendorf D, Kefford RF, Grob JJ, et al: Phase II trial (BREAK-2) of the BRAF inhibitor dabrafenib (GSK2118436) in patients with metastatic melanoma. J Clin Oncol 31: 3205-3211, 2013.

15. Budwit-Novotny DA, McCarty KS, Cox EB, Soper JT, Mutch DG, Creasman WT, Flowers JL and McCarty KS Jr: Immunohistochemical analyses of estrogen receptor in endometrial adenocarcinoma using a monoclonal antibody. Cancer Res 46: 5419-5425, 1986.

16. Specht E, Kaemmerer D, Sänger J, Wirtz RM, Schulz S and Lupp A: Comparison of immunoreactive score, HER2/neu score and $\mathrm{H}$ score for the immunohistochemical evaluation of somatostatin receptors in bronchopulmonary neuroendocrine neoplasms. Histopathology 67: 368-377, 2015.

17. Lei Y, Li HX, Jin WS, Peng WR, Zhang CJ, Bu LJ, Du YY, Ma T and Sun GP: The radiosensitizing effect of Paeonol on lung adenocarcinoma by augmentation of radiation-induced apoptosis and inhibition of the PI3K/Akt pathway. Int J Radiat Biol 89: 1079-1086, 2013.

18. Rygaard K and Spang-Thomsen M: Quantitation and gompertzian analysis of tumor growth. Breast Cancer Res Treat 46: 303-312, 1997.

19. Kocab AJ and Duckett CS: Inhibitor of apoptosis proteins as intracellular signaling intermediates. FEBS J 283: 221-231, 2016.

20. Goldar S, Khaniani MS, Derakhshan SM and Baradaran B: Molecular mechanisms of apoptosis and roles in cancer development and treatment. Asian Pac J Cancer Prev 16: 2129-2144, 2015.

21. Mohammad RM, Muqbil I, Lowe L, Yedjou C, Hsu HY, Lin LT, Siegelin MD, Fimognari C, Kumar NB, Dou QP, et al: Broad targeting of resistance to apoptosis in cancer. Semin Cancer Biol 35 (Suppl): S78-S103, 2015. 
22. Hartman ML and Czyz M: Anti-apoptotic proteins on guard of melanoma cell survival. Cancer Lett 331: 24-34, 2013.

23. Moldoveanu T, Follis AV, Kriwacki RW and Green DR: Many players in BCL-2 family affairs. Trends Biochem Sci 39: 101-111, 2014.

24. Hata AN, Engelman JA and Faber AC: The BCL2 family: Key mediators of the apoptotic response to targeted anticancer therapeutics. Cancer Discov 5: 475-487, 2015.

25. Silke J and Vucic D: IAP family of cell death and signaling regulators. Methods Enzymol 545: 35-65, 2014.

26. Liston P, Fong WG, Kelly NL, Toji S, Miyazaki T, Conte D, Tamai K, Craig CG, McBurney MW and Korneluk RG: Identification of XAF1 as an antagonist of XIAP anti-Caspase activity. Nat Cell Biol 3: 128-133, 2001.

27. Zhao WJ, Deng BY, Wang XM, Miao Y and Wang JN: XIAP associated factor 1 (XAF1) represses expression of X-linked inhibitor of apoptosis protein (XIAP) and regulates invasion, cell cycle, apoptosis, and cisplatin sensitivity of ovarian carcinoma cells. Asian Pac J Cancer Prev 16: 2453-2458, 2015.
28. Zhu LM, Shi DM, Dai Q, Cheng XJ, Yao WY, Sun PH, Y, Qiao MM, Wu YL, Jiang SH, et al: Tumor suppressor XAF1 induces apoptosis, inhibits angiogenesis and inhibits tumor growth in hepatocellular carcinoma. Oncotarget 5: 5403-5415, 2014.

29. Ju WC, Huang GB, Luo XY, Ren WH, Zheng DQ, Chen PJ, Lou YF and Li B: X-linked inhibitor of apoptosis-associated factor 1 (XAFl) enhances the sensitivity of colorectal cancer cells to cisplatin. Med Oncol 31: 273, 2014.

30. Huang J, Yao WY, Zhu Q, Tu SP, Yuan F, Wang HF, Zhang YP and Yuan YZ: XAF1 as a prognostic biomarker and therapeutic target in pancreatic cancer. Cancer Sci 101: 559-567, 2010.

31. Ng KC, Campos EI, Martinka M and Li G: XAF1 expression is significantly reduced in human melanoma. J Invest Dermatol 123: 1127-1134, 2004 